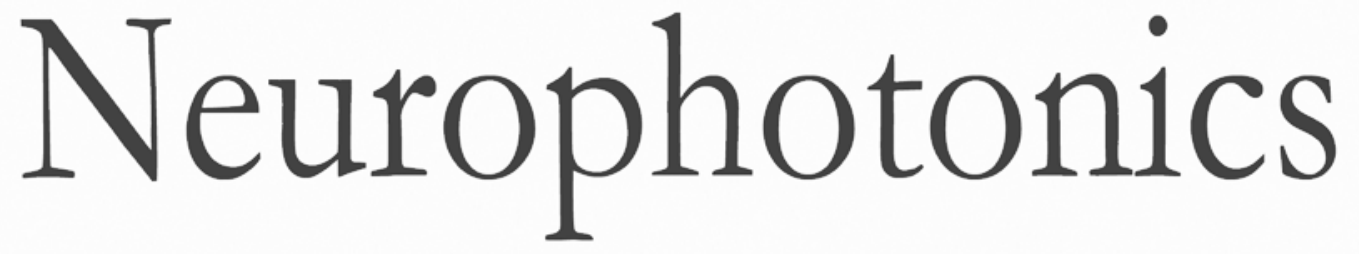

\title{
Which is more costly in Chinese to English simultaneous interpreting, "pairing" or "transphrasing"? \\ Evidence from an fNIRS neuroimaging study
}

Xiaohong Lin

Victoria Lai Cheng Lei

Defeng Li

Zhen Yuan 


\title{
Which is more costly in Chinese to English simultaneous interpreting, "pairing" or "transphrasing"? Evidence from an fNIRS neuroimaging study
}

\author{
Xiaohong Lin, ${ }^{\mathrm{a}, \mathrm{b}}$ Victoria Lai Cheng Lei, ${ }^{\mathrm{c}, \dagger}$ Defeng $\mathrm{Li}^{\mathrm{c}}{ }^{\mathrm{c}, *}$ and Zhen Yuan ${ }^{\mathrm{a}, *}$ \\ aUniversity of Macau, Bioimaging Core, Faculty of Health Sciences, Macao, China \\ ${ }^{b}$ Hangzhou Normal University, Institutes of Psychological Sciences, Hangzhou, China \\ 'University of Macau, Centre for Studies of Translation, Interpreting and Cognition, Faculty of Arts and Humanities, Macao, China
}

\begin{abstract}
This study examined the neural mechanism underlying two translation strategies associated with Chinese to English simultaneous interpreting (SI) targeting the left prefrontal cortex (PFC), which is generally involved in the control of interference and conflict resolution and has been identified as the brain area that plays a pivotal role in SI. Brain activation associated with the two strategies including "pairing" and "transphrasing" were compared with that from "nontranslation," which keeps the source language item unchanged in the target language production and is considered as a tactic that does not require complex cognitive operation associated with bilingual processing effort. Our findings revealed that "pairing" elicited the strongest and almost immediate brain activation in the Broca's area, and "transphrasing" resulted in the most extensive and strongest activation overall in the left PFC. By contrast, "nontranslation" induced very little brain activation in these regions. This work, which represents one of the first efforts in investigating brain activation related to translation strategies involving different levels of cognitive control, will not only pave a new avenue for better understanding of the cognitive mechanism underlying SI but also provide further insight into the role that the Broca's region plays in domaingeneral cognitive control. ๑ 2018 Society of Photo-Optical Instrumentation Engineers (SPIE) [DOI: 10.1117/1.NPh.5.2.025010]
\end{abstract}

Keywords: functional near-infrared spectroscopy; translation strategies; simultaneous interpreting.

Paper 17135RR received Nov. 29, 2017; accepted for publication May 11, 2018; published online Jun. 5, 2018.

\section{Introduction}

As one of the most complex and demanding language tasks, which involves listening to a speech uttered in a source language (SL) and translating it into a target language (TL) at the same time, professional simultaneous interpreting (SI) requires a large amount of cognitive capacity and a wide range of cognitive abilities associated with language processing across two languages, including decision making (e.g., choosing an SI strategy) and executive functions, such as working memory, inhibition control, and cognitive flexibility. ${ }^{1-7}$ Identifying the neuromarker of SI is essential for better understanding the neural mechanism of extreme language control. To the best of our knowledge, no neuroimaging study has been performed to examine the neural correlates of particular translation strategies. Consequently, the investigation into the brain activation associated with different SI strategies by neuroimaging techniques can aid to reveal the brain cognitive mechanism involved in particular strategies and can also aid to determine the most effective strategy in SI-a mode of interpreting that constantly places the interpreter under extreme time pressure or at risk of cognitive overload. More importantly, the neural correlates of various SI strategies may further provide insight into bilingual processing and cognitive control.

\footnotetext{
*Address all correspondence to: Zhen Yuan, E-mail: zhenyuan@umac.mo; Defeng Li, E-mail: defengli@umac.mo

†Joint first coauthorship.
}

In this study, two primary SI strategies generally adopted by simultaneous interpreters were carefully examined at the lexical level-one through direct associative links and the other through conceptual mediation. ${ }^{8-13}$ In particular, we name the strategy, which "pairs up" translation-equivalent structures between SL and TL stored in long-term memory as "pairing." The other strategy is "transphrasing," which is meaning based and involves a "bottom-up" monolingual processing in the SL, a nonverbal conceptual level and then a "top-down" monolingual processing in the TL. For example, in the context of Chinese to English interpreting, "fu ling" in the Chinese ST can be rendered into "Poria" by "pairing," or into "a Chinese herbal medicine" by "transphrasing."

Referring to the activation threshold theory, ${ }^{14}$ Paradis $^{8}$ hypothesized that when a bilingual person speaks one language, the activation threshold of the nonselected language is raised sufficiently to prevent interference. In SI, the activation thresholds of both working languages of the professional interpreter have to be lowered so that both language systems can be engaged concurrently. Importantly, De Groot ${ }^{13}$ demonstrated that during an act of translation, translation-equivalent structures in memory are activated in "close temporal proximity," and consequently, they can be linked up with one another. Such memory pairs can also be acquired through conscious "paired-associate learning." 13 Glossary building, an active process of obtaining the memory pairs, is considered an important component in professional interpreting practice. ${ }^{15}$

$2329-423 X / 2018 / \$ 25.00$ @ 2018 SPIE 
In addition, "transphrasing" refers to a hybrid term named after "translating" and "paraphrasing." 16 De Groot ${ }^{13}$ hypothesized that taking the conceptually mediated route, the interpreter exploited "the same comprehension and production apparatus as used in monolingual language tasks." The speech input is first processed "upward" through the SL system, followed by a "peripheral analysis of the input" that leads to the formation of a "nonverbal conceptual representation" of the input. Then, there is a "downward process" that begins with this conceptual representation and concludes with the articulation of the conceptual representation in the TL. Paradis ${ }^{8}$ suggested that unlike "pairing," which underlies professionalism, "transphrasing" is likely to be used by bilinguals, who have not yet acquired the expertise in translation, exhibiting that lay translators generally find it hard to produce translation equivalents that they have no problem using in monolingual settings. The assumption is that the SL form in short-term memory may inhibit access to its translation equivalent, and such inhibition is actually essential for bilinguals to prevent interference of the nonselected language in monolingual contexts.

Furthermore, previous reports indicate that when performing the complex and demanding task of SI, having the equivalent items across the SL and TL certainly can maintain the quality of SI and reduce the mental effort of the interpreter. ${ }^{8,17-19}$ Meanwhile, "transphrasing" involves full comprehension and often wordy outputs, which demands more processing effort of the simultaneous interpreter. ${ }^{8,13,17,20-23}$ This strategy is not exclusively used by lay interpreters. Professional interpreters may resort to it when they understand a term in the SL, but do not know the translation equivalent in the TL. For example, the French word "tableur" was rendered "the programme which defines rows and columns and allows calculations to be made" at a conference in the 1980s when the interpreter obviously did not know "spreadsheet," its translation equivalent in English. Gile ${ }^{17}$ demonstrated that this tactic, while "efficient informationally," requires a significant amount of time and processing capacity.

Meanwhile, interpreting by "pairing" may be swift and result in precise and concise outputs. However, from the perspective of language control, ${ }^{11,24,25}$ as it involves activating translation equivalents across two languages concurrently while suppressing the item in the nonselected language in the production, the effort required for language control may be extremely intense. By contrast, "transphrasing" may take more time and result in cumbersome outputs. However, as it first goes through the loop of decoding in the SL till a nonverbal concept is formed, followed by encoding in the TL, consequently, the interpreter adopting this strategy is less exposed to interferences between the two languages. ${ }^{26}$ Thus, "transphraising" may require less resources for inhibition in the production in comparison with "pairing."

In addition to the two major SI strategies, there exists another SI strategies acceptable in certain situations- "nontranslation," 27 which means repeating SL expressions in the delivery rather than translating them. For example, "fu ling" in the Chinese input can be rendered into "fu ling" in the English output by "nontranslation." Gile ${ }^{17}$ reported that when professional interpreters encounter proper names or technical terms they do not know, they are likely to repeat the SL sound of the term in the delivery. It has been claimed to be an effort-reduction tactic that does not call for complex cognitive operation. ${ }^{17}$ Previous work also showed that bilinguals do access both languages even when they are in monolingual modes. ${ }^{6,11,24,28,29}$ It will be useful to use "nontranslation" as the baseline against "pairing" and "transcoding." In the context of SI, "nontranslation" inevitably needs to engage both languages and the interpreters also need a cognitive mechanism that prevents interference from the non-TL, ${ }^{30}$ although we may assume that the effort required for activation and inhibition in the production is less intense.

Interestingly, functional neuroimaging technologies make themselves ideal candidates for measuring the cognitive effort involved in the translation strategies mentioned above. Various functional neuroimaging studies were conducted to inspect the brain activation involved in SI. ${ }^{2,5,31,32}$ More importantly, it has been discovered that the left prefrontal cortex (PFC) including the Broca's area is linked to lexical search, semantic processing, bilingual processing, verbal working memory, and control of interference and conflict resolution. ${ }^{31-36}$

In this study, functional near-infrared spectroscopy (fNIRS) was utilized to explore how the translation strategies in SI are associated with the hemodynamic responses in the left PFC, including the Broca's area, which has been established as a region playing an essential role in the production of speech, ${ }^{37-44}$ language switching, ${ }^{45}$ and cognitive control. ${ }^{46-48}$ In particular, the brain activation in the left PFC elicited, respectively, by "pairing" and "transphrasing" during the Chinese to English SI was compared with that with "nontranslation." fNIRS, a portable and noninvasive functional brain imaging technique, requires very few body constraints, exhibiting the potential to allow more ecologically valid investigations-the subjects can sit, stand, and even walk during the tests. ${ }^{49,50}$ Compared to fNIRS, fMRI and PET have certain disadvantages, which required the participants to lie absolutely still during the scans and therefore cannot approximate the actual SI working conditions. In addition, fNIRS is able to offer the quantitative hemodynamic measures for both oxyhemoglobin $(\mathrm{HbO})$ and deoxyhemoglobin $(\mathrm{HbR})$, which is essential for revealing the rapid changes of dynamic patterns in the brain, including the changes of blood oxygen, blood volume, and blood flow. ${ }^{51-55}$

Combining behavioral measures and functional neuroimaging techniques, this study aims to examine the cognitive effort associated with "pairing," "transphrasing," and "nontranslation" during Chinese to English SI. As such, we hypothesize that: (a) compared with "transphrasing," "pairing" takes up less effort for bilingual language processing (decoding and encoding) but requires more effort for cognitive control (control of interference); (b) "transphrasing" takes up more effort for bilingual language processing (decoding and encoding) but requires less effort for cognitive control (control of interference); and (c) among the three strategies, "nontranslation" requires the least language processing and control effort. This study will definitely pave an avenue for an improved understanding of the cognitive mechanism underlying the Chinese to English SI.

\section{Methods}

\subsection{Participants}

Ten postgraduate students (four males, mean age $=24$ years, S.D. $=2.21$ years) majoring in translation studies at the University of Macao (UM) participated in this study. All participants were native Chinese (Mandarin) speakers and became fluent in English after age 12. All participants had a high proficiency in written and spoken English (TEM-8, the highest level for English major students in the Chinese mainland tertiary education system). All participants were right handed with 
normal or correction-to-normal vision. None of them had reported histories of neurological or psychiatric disorders. All participants gave written informed consent in accordance with the Declaration of Helsinki. The protocol was approved by the Ethics Committees of University of Macau.

\subsection{Stimuli}

In SI practice, "nontranslation" does not really function at sentence level, and the use of "nontranslation" should be mostly restricted to proper names and technical terms. Also, "memory pairs" at the sentence level are rare. Furthermore, translating a whole sentence usually involves more than one strategy. If we use sentences instead of words, the participants may adopt two or more strategies to do the SI. Consequently, for the sake of controlling variables, the stimulus materials were all two-character cultural-specific items selected from the UM
Magazine (Chinese version). The corpus contains totally 159,058 characters, 3983 common nouns, and 1869 culture-specific items. From the culture-specific items in the corpus, 15 were selected for task one, 15 for task two, and 15 for task three, which were to be translated by pairing, transphrasing, and nontranslation, respectively. There was no distinguishing feature between high-frequency items and low-frequency items in the selection, as the corpus is relatively small and it was expected that subjects had been exposed to the culture-specific items in the corpus. Trial run results also confirmed that the stimuli selected from the corpus, regardless of frequency, were familiar to the subjects.

\subsection{Experimental Design}

The subjects were asked to perform three tasks during the experiment, namely a pairing task, a nontranslation task, and

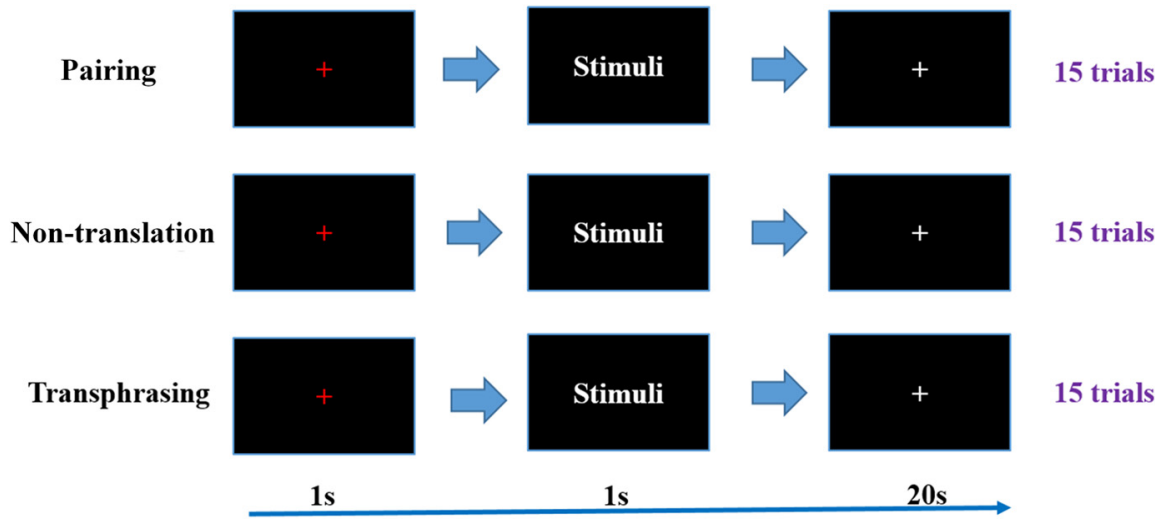

Fig. 1 Schematic of the experimental design

(a)

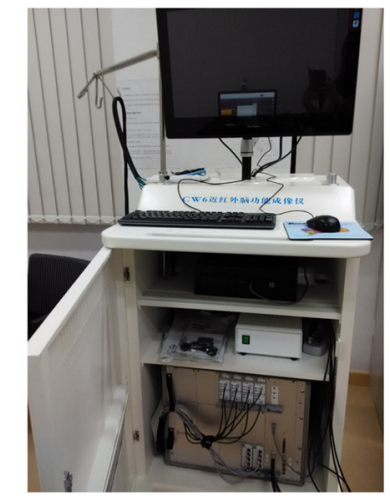

(c) (b)

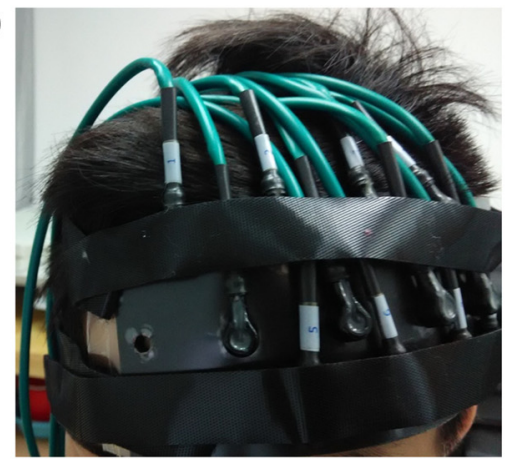

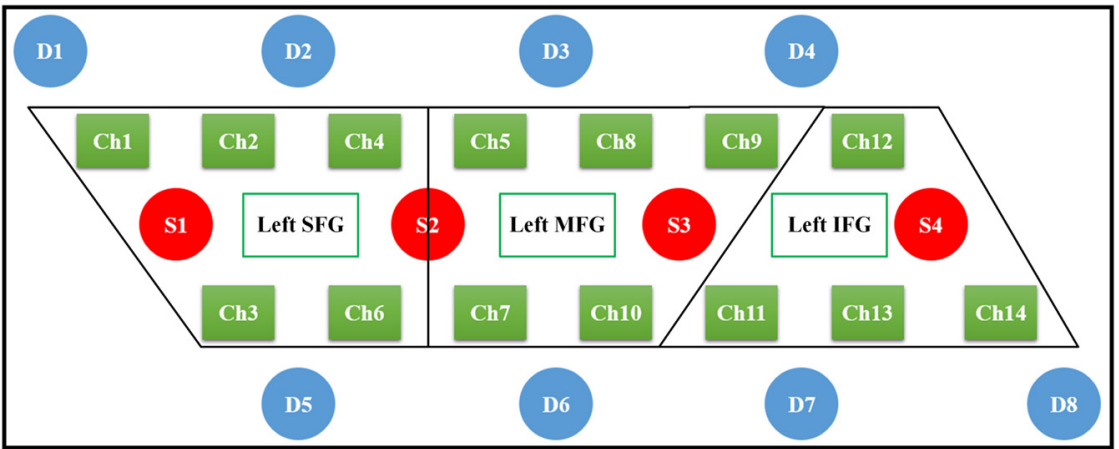

Fig. 2 (a) The CW6 fNIRS system. (b) The head patch covering the frontal region specially designed for the experimental tests of present study. (c) The configurations of sources, detectors, and channels. The red dots denote the sources, the blue dots represent the detectors, and the green dots define the channels. 
a transphrasing task. Each task included 15 trials and each trial lasted 22s, which included a prestimulus period of $1 \mathrm{~s}$ with a red fixation cross presented at the screen centre of the monitor, a stimulus period of $1 \mathrm{~s}$, and then a poststimulus and recovery period of $20 \mathrm{~s}$ with a white fixation cross displayed at the screen centre of the monitor (Fig. 1). It took about 20 min to finish the data acquisition.

Task 1: Participants were instructed to translate orally the two-character culture-specific items presented on the computer screen into English. The subjects were not informed which strategy to use. However, it was expected that as students of UM, they should be familiar with the expressions in both Chinese and English. It was also predicted that as competent bilinguals who had received training in SI, they would have access to the translation equivalent in the TL. Trial run results validated that items in task 1 were likely to yield "pairing," and subjects claimed at post-hoc interviews that they automatically rendered the items by "pairing" as the translation equivalents in the TL were known to them. For example, "fu ling" in the Chinese input can be rendered into "Poria" in the English output by "pairing."

Task 2: Participants were instructed to orally translate the two-character culture-specific items presented on the computer screen into English using the "nontranslation" strategy, i.e., producing the sound of the SL item rather than giving its direct equivalent in the TL. For example, "fu ling" in the Chinese input can be rendered into "fu ling" in the English output by "nontranslation."

Task 3: Participants were instructed to translate orally the two-character culture-specific items presented on the monitor screen into English by using the "transphrasing" strategy, i.e., explaining what/where the item is rather than giving its direct equivalent in the TL. For example, "fu ling" in the Chinese input can be rendered into "a Chinese herbal medicine" by "transphrasing."

\section{4 fNIRS Data Recordings}

The experiments were conducted by using a continuous wave (CW) fNIRS system [Fig. 2(a)] with four laser sources and eight optical detectors (CW6 fNIRS system; TechEn Inc., Milford, Massachusetts). This system with two CW lights at wavelengths 690 and $830 \mathrm{~nm}$ is able to detect the changes in both $\mathrm{HbO}$ and $\mathrm{HbR}$ concentrations in the human brain. For the present study, the fNIRS optodes were placed on a homemade plastic patch $(6 \mathrm{~cm} \times 18 \mathrm{~cm})$ covering the left PFC (region of interest). Two nylon bands were used to keep the patch attached to the scalp. The configurations of the source and detector pairs, which consisted of 14 channels covering the left PFC, are displayed in Figs. 2(b) and 2(c).$^{56}$ The distance between each source and each detector was $3 \mathrm{~cm}$ and the fNIRS sampling rate was kept at $50 \mathrm{~Hz}$. To reduce the effect of physiology noise and instrumental noise to the greatest extent, the data were processed by a bandpass filter of a high cut-off filter at $0.2 \mathrm{~Hz}$ and a low cut-off filter at $0.01 \mathrm{~Hz}$. The high cut filter can remove the high-frequency measurement instrumental noise while the low cut filters can remove the slow physiological noise. $^{54}$

In addition, a three-dimensional (3-D) magnetic space digitizer Patriot Digitizer (Polhemus Inc.) was utilized to capture the 3-D spatial coordinates of each optode placed on the participant's scalp. A probabilistic registration method from NIRSSPM software was used to estimate each channel's corresponding coordinates in the Montreal Neurological Institute (MNI)
Table 1 The mean MNI coordinates from all subjects and associated brain regions for the 14 channels.

\begin{tabular}{|c|c|c|c|c|c|}
\hline Channels & $x$ & $y$ & $z$ & Brain regions & Probability \\
\hline Ch1 & -8 & 63 & 35 & $\begin{array}{l}\text { Left superior frontal } \\
\text { gyrus (BA10) }\end{array}$ & 1 \\
\hline Ch2 & -14 & 61 & 36 & $\begin{array}{l}\text { Left superior frontal } \\
\text { gyrus (BA9) }\end{array}$ & 1 \\
\hline Ch3 & -19 & 72 & 10 & $\begin{array}{l}\text { Left superior frontal } \\
\text { gyrus (BA10) }\end{array}$ & 0.99 \\
\hline Ch4 & -25 & 58 & 33 & $\begin{array}{l}\text { Left superior frontal } \\
\text { gyrus (BA46) }\end{array}$ & 0.64 \\
\hline Ch5 & -34 & 52 & 31 & $\begin{array}{l}\text { Left middle frontal } \\
\text { gyrus (BA46) }\end{array}$ & 0.93 \\
\hline Ch6 & -29 & 67 & 7 & $\begin{array}{l}\text { Left superior frontal } \\
\text { gyrus (BA10) }\end{array}$ & 0.76 \\
\hline Ch7 & -38 & 62 & 6 & $\begin{array}{l}\text { Left middle frontal } \\
\text { gyrus (BA10) }\end{array}$ & 0.81 \\
\hline Ch8 & -42 & 45 & 32 & $\begin{array}{l}\text { Left middle frontal } \\
\text { gyrus (BA46) }\end{array}$ & 1 \\
\hline Ch9 & -47 & 35 & 33 & $\begin{array}{l}\text { Left middle frontal } \\
\text { gyrus (BA45) }\end{array}$ & 0.74 \\
\hline Ch10 & -45 & 54 & 5 & $\begin{array}{l}\text { Left middle frontal } \\
\text { gyrus (BA46) }\end{array}$ & 0.77 \\
\hline Ch11 & -52 & 44 & 4 & $\begin{array}{l}\text { Left inferior frontal } \\
\text { gyrus (BA45) }\end{array}$ & 0.87 \\
\hline Ch12 & -54 & 22 & 31 & $\begin{array}{l}\text { Left inferior frontal } \\
\text { gyrus (BA44) }\end{array}$ & 0.73 \\
\hline Ch13 & -56 & 33 & 3 & $\begin{array}{l}\text { Left inferior frontal } \\
\text { gyrus (BA45) }\end{array}$ & 1 \\
\hline Ch14 & -57 & 17 & 2 & $\begin{array}{l}\text { Left inferior frontal } \\
\text { gyrus (BA48) }\end{array}$ & 0.70 \\
\hline
\end{tabular}

space, ${ }^{57}$ and the results were provided in Table 1 . The 3-D spatial coordinates of 12 optodes and 14 channels along the cortex were plotted and illustrated in Figs. 3(a) and 3(b).

\subsection{Data Analysis}

The fNIRS data preprocessing were performed using Homer2_UI (v1.5.2). ${ }^{58}$ The raw data were first converted to optical density changes, and then converted to oxyhemoglobin $(\mathrm{HbO})$ and deoxyhemoglobin $(\mathrm{HbR})$ concentration changes at different time points using the modified Beer-Lambert Law. ${ }^{59}$ The generated continuous data of $\mathrm{HbO}$ and $\mathrm{HbR}$ were further processed by a low cut-off filter of $0.2 \mathrm{~Hz}$ and subsequently a high cut-off filter of $0.015 \mathrm{~Hz}$. An automatic motion artifacts detection algorithm from Homer2 fNIRS processing package were utilized for motion correction. ${ }^{58}$ The duration of each trial was $21 \mathrm{~s}$, which included a 1-s prestimulus period and a 20-s stimulus and recovery period. As it has been widely recognized that the change in $\mathrm{HbO}$ concentration is the most sensitive indicator of hemodynamic responses, only $\mathrm{HbO}$ data were analyzed in this study. ${ }^{60}$ After several trials with obvious 


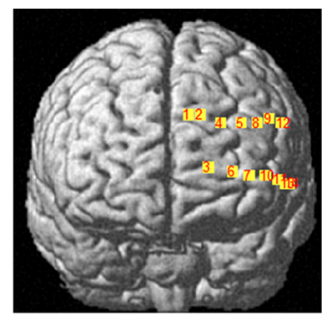

(a1)

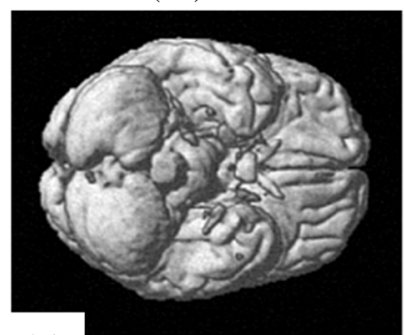

(b)

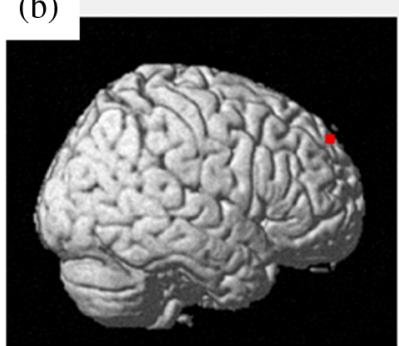

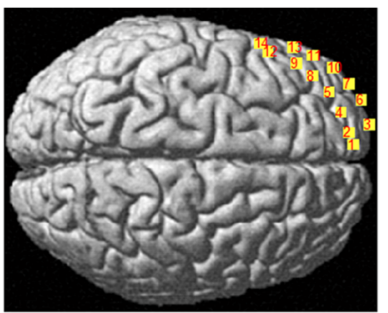

(a2)
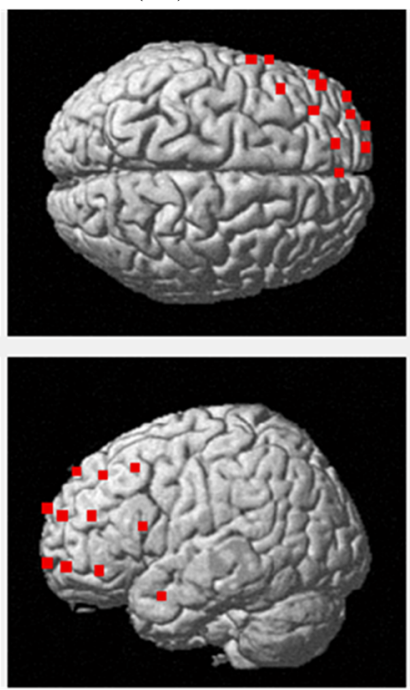

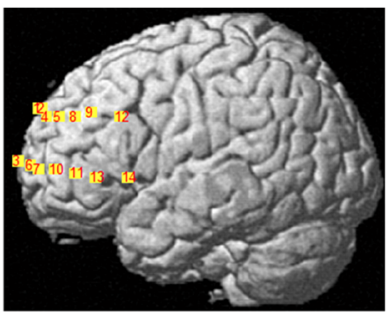

(a3)
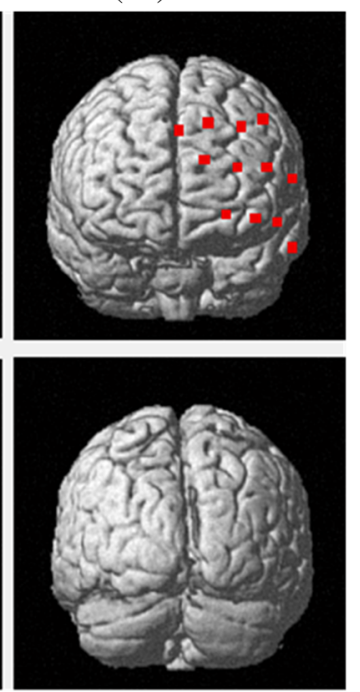

Fig. 3 (a1)-(a3) The estimated locations of the 14 fNIRS channels placed along the cortex with different views. (b) The estimated cortical locations of the four sources and eight detectors.

translation mistakes discarded, the run average of $\mathrm{HbO}$ concentration was calculated, and then the grand-averaged $\mathrm{HbO}$ signals from 10 subjects under the three conditions were generated. Finally, the peak amplitude of each channel from each participant during the stimulus period was extracted for further statistical analysis. All $p$ values of $F$-test were corrected by false-discovery rate (FDR, $p<0.05) .{ }^{61}$ Relationships between behavior data and $\mathrm{HbO}$ signals were also generated by Pearson correlation analysis, which measures the strength and direction of the linear relationship between two variables, describing the direction and degree to which one variable is linearly related to another. All statistical analyses were conducted with SPSS 20.0.

\section{Results}

\subsection{Behavioral Results}

The mean translation accuracy for each participant was calculated. A repeated measures ANOVA was performed, with the mean accuracy as the within-subject variable (stimulus types: pairing, transphrasing, and nontranslation). We discovered that the main effects reached the conventional level of significance $\left(F=12.316, p<0.0001, \eta^{2}=0.57\right)$. Further analysis showed that the accuracy of transphrasing task $(M=12.9$, $\mathrm{SD}=1.729)$ was lower than that of the pairing task $(M=14.7$, $\mathrm{SD}=0.675.), p=0.005$. In addition, the accuracy of transphrasing task was also lower compared to that of the nontranslation task $(M=14.9, \mathrm{SD}=0.316), p=0.005$. The behavior analysis results were provided in Fig. 4.

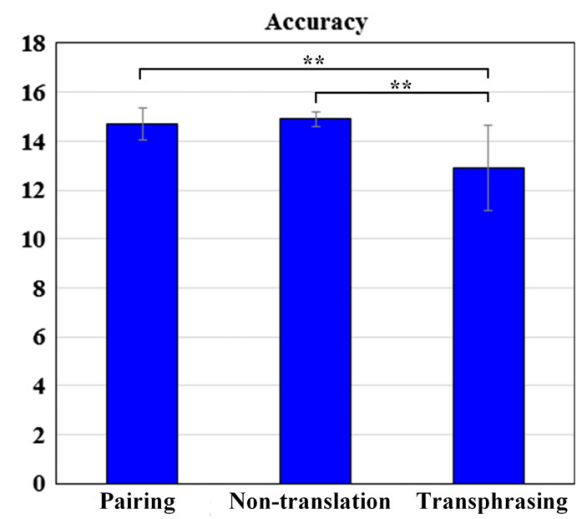

Fig. 4 The mean accuracy of the three tasks based on analysis of the behavioral data of the study $\left(p<0.01^{* *}\right)$.

\subsection{Neuroimaging Results by Using fNIRS}

According to the recordings of event-related translation tasks from 10 subjects, the grand average of $\mathrm{HbO}$ concentration change was calculated for each channel. Figure 5 displayed the time courses of concentration changes in $\mathrm{HbO}$ for each of the three tasks with associated channels. It was discovered that for all the three tasks, there was a task-related increase in the concentration change of $\mathrm{HbO}$ several seconds after the onset of the triggers. Once the concentration change in $\mathrm{HbO}$ reached the peak, it returned to the baseline again. We also discovered that the three tasks exhibited obvious difference in brain hemodynamic responses. For example, the increase in $\mathrm{HbO}$ concentration (peak value) associated with transphrasing was higher 

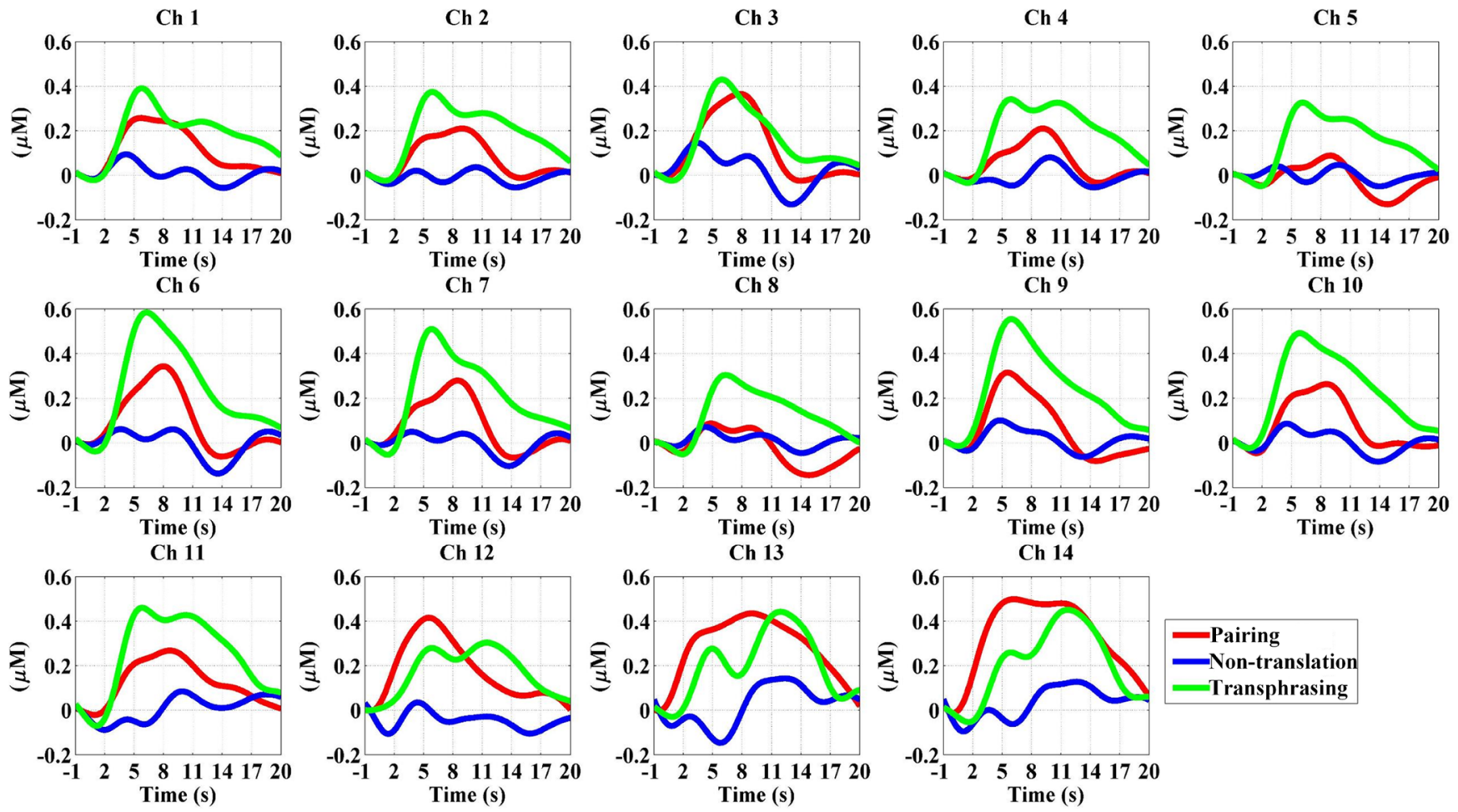

Fig. 5 The time courses of the grand-averaged hemodynamic changes $(\mathrm{HbO})$ associated with, pairing, nontranslation, and transphrasing, which are represented by the red, blue, and green curves, respectively.

than those with pairing and nontranslation across most of the channels. Nontranslation elicited the lowest hemodynamic change for most of the channels compared to transphrasing and pairing.

In addition, a repeated measures ANOVA was also performed by using the peak value of the $\mathrm{HbO}$ concentration change from each channel as the within-subject variable (stimulus types: pairing, transphrasing, and nontranslation). Significant main effects in channels $1-10\left(F s \geq 12.048, p s<0.0001, \eta^{2} s \geq 0.572\right)$ and channel $14\left(F(2,18)=4.005, p=0.046, \eta^{2}=0.308\right)$ were identified with FDR correction. Further statistical analysis exhibited that the pairing stimuli elicited significantly higher peak values of hemodynamic responses than the nontranslation stimuli in channels $1,3,6,10$, and 14 ( $p s \leq 0.03$ ). Meanwhile, the transphrasing stimuli elicited significantly higher peak values than the nontranslation stimuli in channels $1-10$ ( $p s \leq 0.009$ ). Likewise, the transphrasing stimuli also elicited significantly higher peak values than the pairing stimuli in channels $1-10$ ( $p s \leq 0.015)$. The statistical analysis results were provided in Table 2 .

\subsection{Relationship Between Behavior Data and $\mathrm{HbO}$ Signals}

The Pearson correlation analysis was performed between the behavior results (ACC) and the peak value of the $\mathrm{HbO}$ concentration change from each channel for all three SI strategies. Statistical analysis results in Table 3 showed that significant correlations were identified in channels $1,2,9,10$ for pairing strategy case, and in channel 8 for transphrasing strategy case. However, the nontranslation strategy case did not exhibit significant correlation.
Table 2 Statistical analysis results. For the mean value, " $p$ " represents pairing, " $n$ " denotes nontranslation, and " $t$ " represents transphrasing.

\begin{tabular}{lccccc} 
Channel & $F$ & $\eta^{2}$ & Mean $(p)$ & Mean $(n)$ & Mean $(t)$ \\
\hline Ch1 & $27.915^{\star *}$ & 0.756 & 0.340 & 0.215 & 0.526 \\
Ch2 & $30.189^{\star *}$ & 0.770 & 0.296 & 0.187 & 0.508 \\
Ch3 & $20.497^{\star *}$ & 0.695 & 0.402 & 0.213 & 0.692 \\
Ch4 & $21.072^{\star *}$ & 0.701 & 0.284 & 0.195 & 0.510 \\
Ch5 & $15.41^{\star *}$ & 0.631 & 0.228 & 0.185 & 0.460 \\
Ch6 & $18.86^{\star *}$ & 0.677 & 0.397 & 0.242 & 0.636 \\
Ch7 & $15.822^{\star *}$ & 0.637 & 0.441 & 0.321 & 0.697 \\
Ch8 & $12.048^{\star *}$ & 0.572 & 0.174 & 0.197 & 0.401 \\
Ch9 & $12.057^{\star *}$ & 0.573 & 0.408 & 0.279 & 0.655 \\
Ch10 & $15.392^{\star *}$ & 0.631 & 0.354 & 0.237 & 0.583 \\
Ch14 & $4.005^{*}$ & 0.308 & 0.734 & 0.364 & 0.564 \\
\hline
\end{tabular}

${ }^{*} p<0.05$

${ }^{\star *} p<0.001$

\subsection{Spatial Mapping of the Brain Activation}

To map the brain activation during the performance of different stimuli-evoked tasks, the $\mathrm{HbO}$ images were also visualized on a brain cortex template, as plotted in Fig. 6. 
Table 3 The correlation between behavior (ACC) data and $\mathrm{HbO}$ signals.

\begin{tabular}{lccc}
\hline Channels & Pairing & Nontranslation & Transphrasing \\
\hline 1 & $-0.727^{*}$ & 0.077 & -0.432 \\
2 & $-0.81^{*}$ & -0.094 & -0.34 \\
3 & -0.209 & 0.031 & -0.067 \\
4 & 0.231 & 0.001 & -0.071 \\
5 & 0.432 & -0.06 & 0.428 \\
6 & 0.425 & -0.456 & -0.455 \\
7 & 0.148 & -0.113 & -0.473 \\
8 & 0.448 & -0.109 & $0.724^{*}$ \\
9 & $-0.762^{*}$ & -0.182 & 0.387 \\
10 & $-0.658^{*}$ & -0.127 & -0.039 \\
11 & 0.091 & 0.001 & 0.242 \\
12 & -0.466 & -0.112 & -0.243 \\
13 & 0.007 & -0.133 & -0.367 \\
14 & 0.362 & -0.212 & -0.159 \\
\hline${ }^{*} p 0.05$ & & &
\end{tabular}

In addition, the grand average of $\mathrm{HbO}$ concentration from each channel was extracted for each time point during the stimulus period, which can be used to describe the brain activation patterns in a dynamic way, as shown in Figs. 7-9.

\section{Discussion}

To the best of our knowledge, this is the first study utilizing neuroimaging techniques to investigate neural correlates of SI strategies. Brain activation in the left PFC associated with the three strategies extensively adopted in SI, namely pairing, nontranslation, and transphrasing, was detected and examined using fNIRS, with the purpose of identifying whether pairing, nontranslation, and transphrasing really involve different levels of bilingual processing efforts conventionally related to SI and different levels of cognitive control. In addition, cognitive efforts involved in the application of the three strategies were also inspected by using behavioral methods.

Consistent with the reports associated with SI practice and training, ${ }^{8,12}$ our findings revealed that the three strategies exhibited obvious differences in terms of behavioral and neuroimaging measurements. The translation accuracy assessed through behavioral measures indicated that the accuracy achieved through transphrasing was significantly lower than that through pairing or nontranslation. With respect to the statistical analysis of the neuroimaging data, we discovered significant difference between these three strategies in terms of $\mathrm{HbO}$ concentration changes. The pairing stimuli elicited significantly higher peak values than the nontranslation stimuli in the left superior frontal gyrus (SFG; channels 1, 3, 6), the left middle frontal gyrus (MFG; channel 10), and the left inferior frontal gyrus (IFG; channel 14). Meanwhile, the transphrasing stimuli

(a)

Pairing
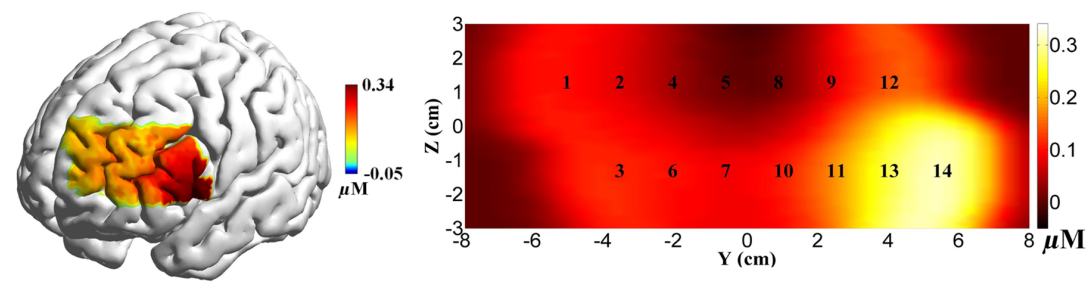

(b)

Non-translation
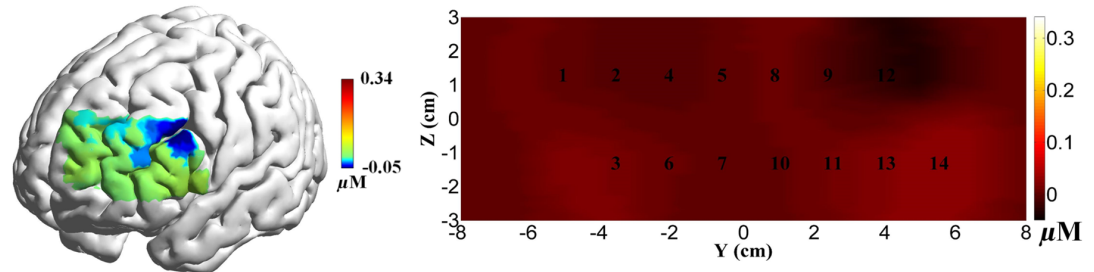

(c)

Transphrasing
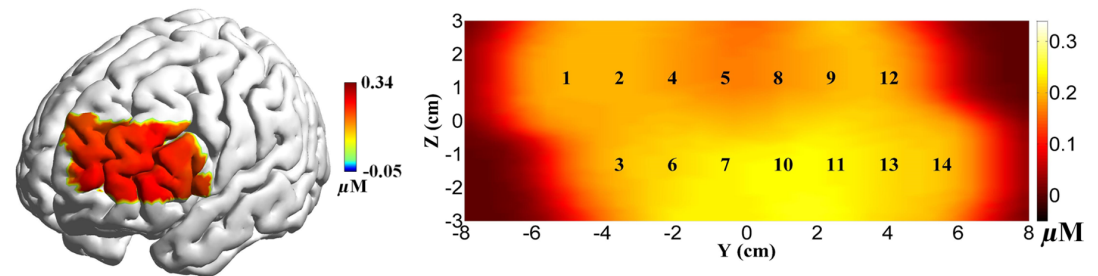

Fig. 6 Grand-averaged $\mathrm{HbO}$ concentration changes associated with (a) pairing, (b) nontranslation, and (c) transphrasing (left: three-dimensional mapping of the brain activation; right: two-dimensional mapping of brain activation). We discovered that the pairing elicited the strongest brain activation in channels 13 and 14, which covered the left IFG, including the Broca's area. Among the three strategies, the nontranslation elicited the lowest brain activation in this region. By contrast, the transphrasing elicited significant activation in all 14 channels, especially in channels 7 and 10, which covered the left MFG, and in channel 11 , which covered the left IFG. 


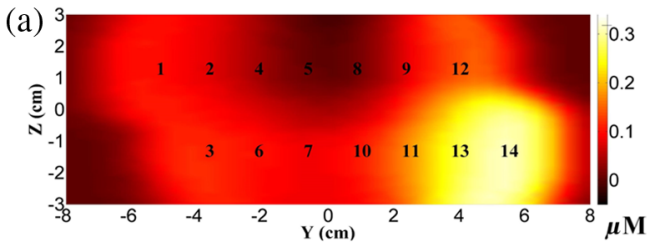

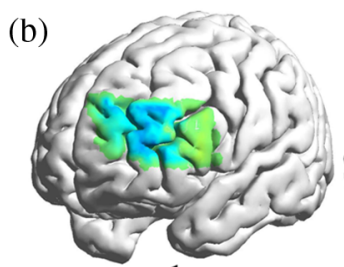

$1 \mathrm{~s}$

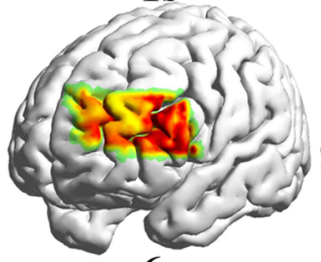

$6 \mathrm{~s}$

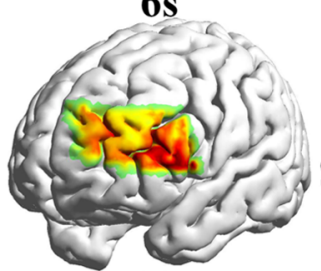

$11 s$

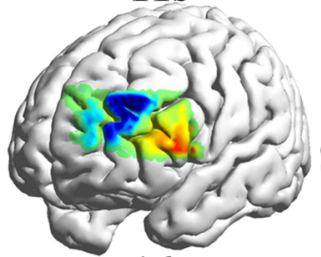

$16 \mathrm{~s}$

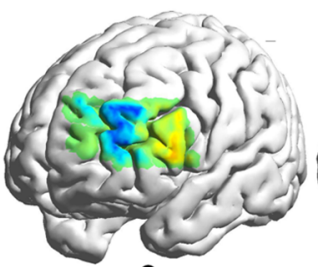

2s

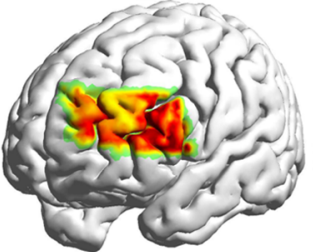

$7 \mathrm{~s}$

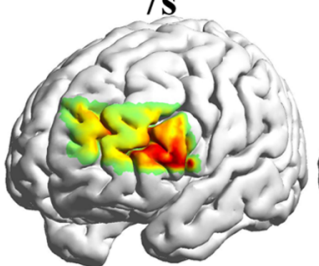

$12 \mathrm{~s}$

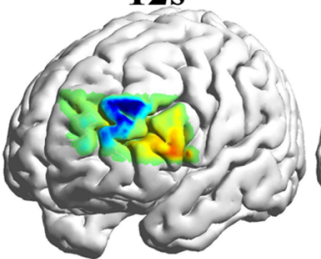

$17 \mathrm{~s}$

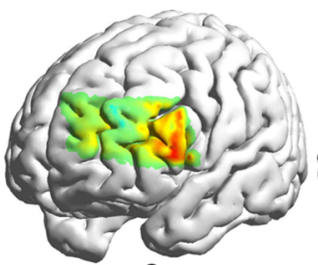

$3 s$

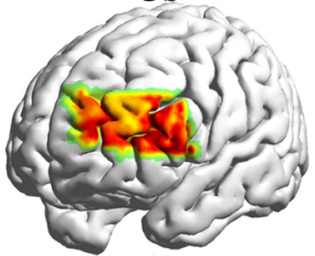

$8 s$

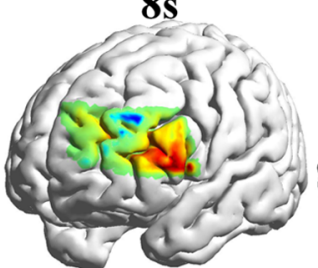

$13 \mathrm{~s}$

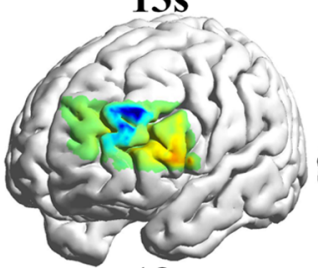

$18 \mathrm{~s}$

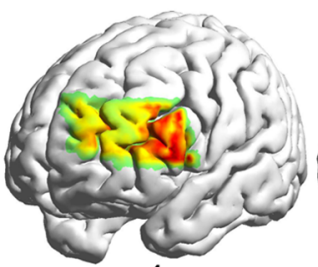

$4 s$

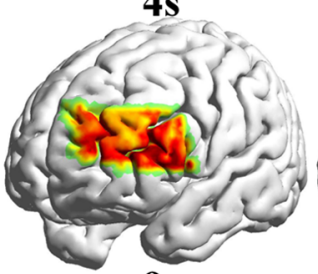

9s

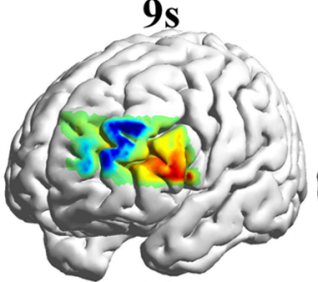

$14 \mathrm{~s}$

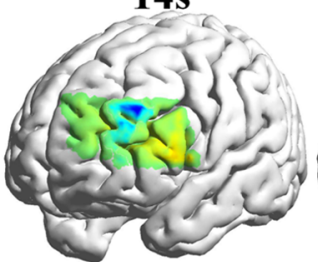

19s

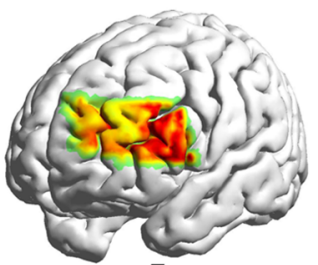

$5 s$

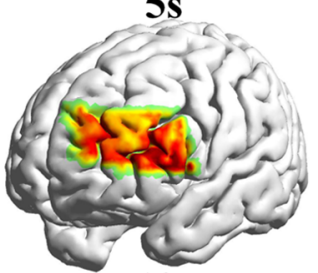

$10 \mathrm{~s}$

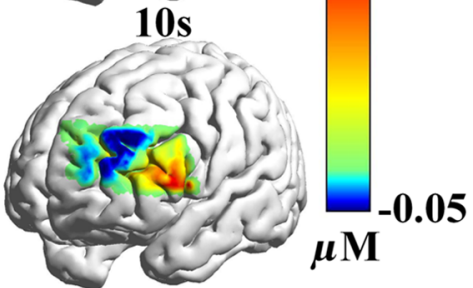

$15 \mathrm{~s}$

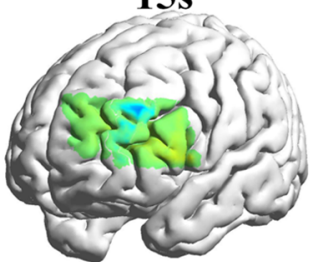

20s

Fig. 7 Brain activation map demonstrating the changes in brain activity over time ( 1 to $20 \mathrm{~s}$ ) associated with pairing stimuli. (a) Mean brain activation across the whole time period. (b) Dynamic brain activation at selected time points. We discovered that $\mathrm{HbO}$ concentration changes in the left IFG [in particular, channels 13 to 14 (Broca's area)] started to increase at $1 \mathrm{~s}$ and continued to grow till $6 \mathrm{~s}$, sustained for several seconds ( 6 to $12 \mathrm{~s}$ ), and then started to decline and the decrease continued all the way to $20 \mathrm{~s}$. On the other hand, $\mathrm{HbO}$ concentration at channel 12 started to increase at $1 \mathrm{~s}$, continued to grow till $6 \mathrm{~s}$ when the decline started, and the decrease continued all the way to $10 \mathrm{~s}$. Interesting brain activities were also detected at channel 3 [the left superior frontal gyrus (SFG)], where the increase in HbO concentration started at $5 \mathrm{~s}$ and the growth continued for several seconds till the decline started at $10 \mathrm{~s}$.

elicited significantly higher peak values than the nontranslation and the pairing cases in the left SFG and the left MFG (channels 1 to 10). The accuracy and the peak of $\mathrm{HbO}$ concentration changes in left SFG (channels 1 to 2) and left MFG (channels 9 to 10) exhibited significant correlation for the paring strategy case, and in the left MFG (channel 8) for the transphrasing strategy case. However, no correlation was identified for the nontranslation strategy case.

Importantly, our optical mapping results based on groupaveraged $\mathrm{HbO}$ concentration changes (Fig. 6) demonstrated that all three strategies induced increased brain activities across the left IFG including Broca's area. In particular, the pairing task generated the highest brain activation in the Broca's area (channels 13-14), the nontranslation task elicited the lowest activation across the whole left PFC (channels 1-14), and the transphrasing strategy induced extensive and enhanced brain activation across the whole left PFC area (all 14 channels).

Specifically, the images in Figs. 7-9 manifested the dynamic brain activation patterns associated with the three strategies. Figure 9 shows that the transphrasing task elicited the highest $\mathrm{HbO}$ concentration in channel 3 (left SFG), channel 6 (left SFG), channel 7 (left MFG), and channel 10 (left MFG) during the period between 6 and $7 \mathrm{~s}$ after the stimuli were presented. Only extremely weak brain activities in the left PFC were detected for the first $3 \mathrm{~s}$. We discovered that the whole left prefrontal region, except for the Broca's area (channels 13 and 14), started to generate increased $\mathrm{HbO}$ concentration at $3 \mathrm{~s}$ and the brain activity continued during the stimuli period from 4 to10 s. It seems the left SFG and the left MFG were the brain regions that were first activated to process semantics during the period 


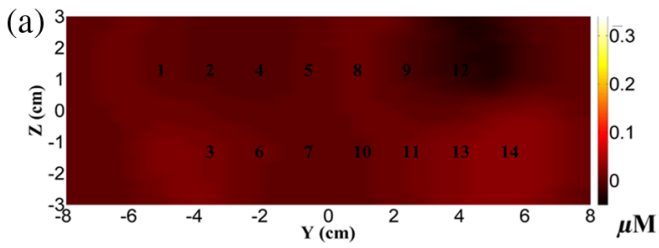

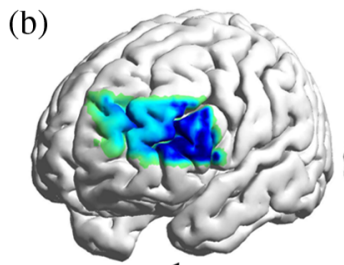

$1 \mathrm{~s}$

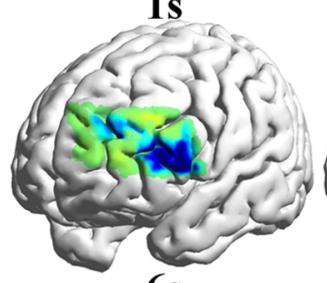

$6 \mathrm{~s}$

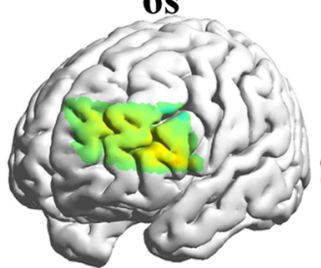

$11 s$

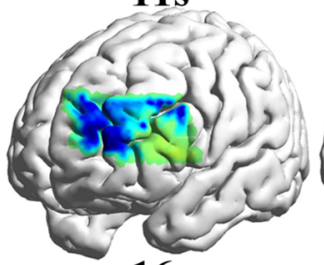

$16 \mathrm{~s}$

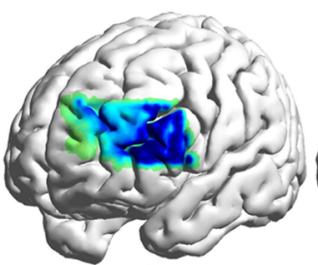

$2 s$

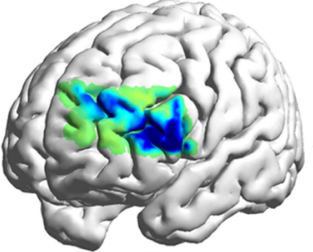

$7 \mathrm{~s}$

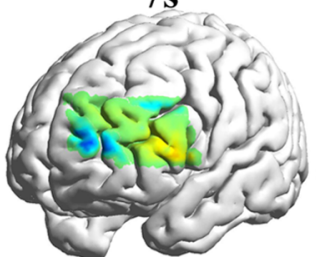

$12 \mathrm{~s}$

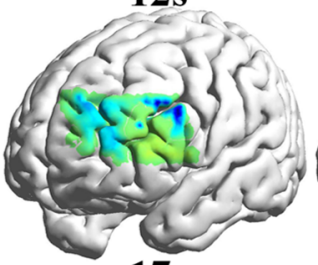

17s

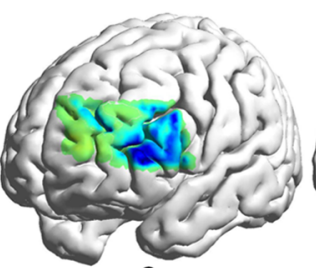

$3 \mathbf{s}$

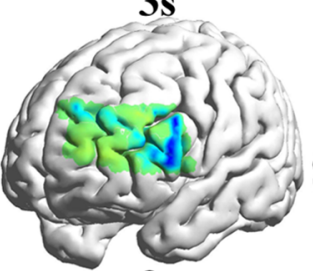

$8 s$

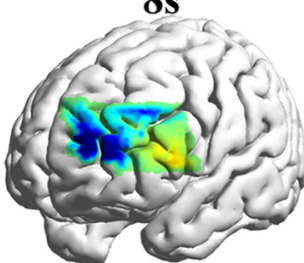

$13 \mathrm{~s}$

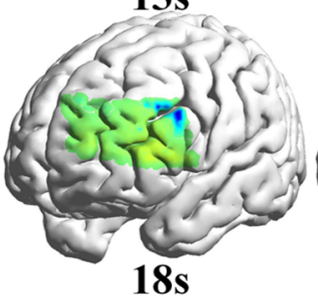

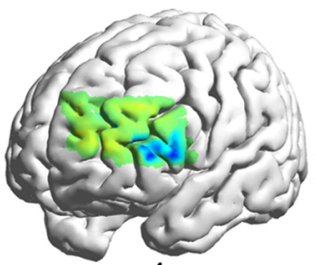

$4 s$

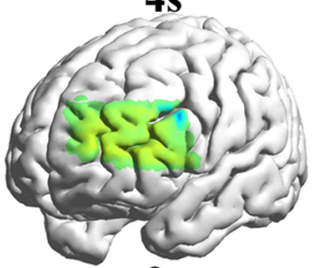

9s

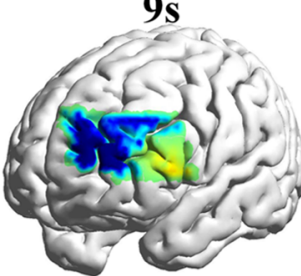

$14 \mathrm{~s}$

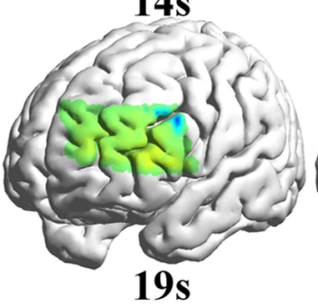

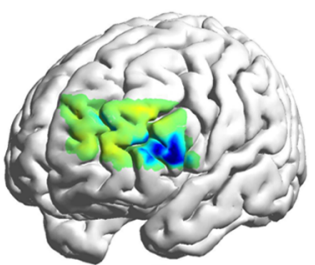

$5 s$

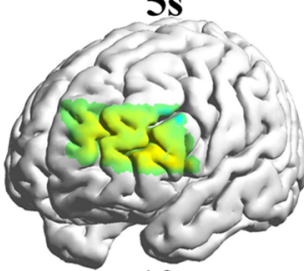

$10 \mathrm{~s}$

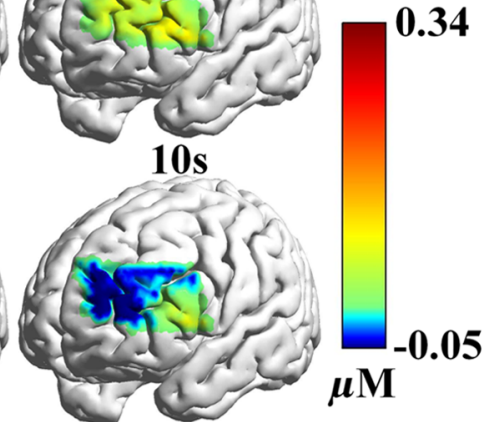

$15 s$

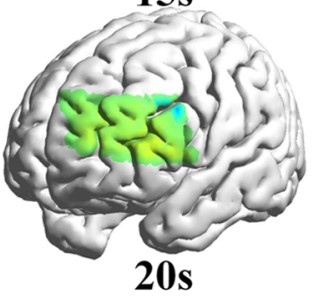

Fig. 8 Brain activation map demonstrating changes in brain activity over time ( 1 to $20 \mathrm{~s}$ ) associated with nontranslation stimuli. (a) Averaged brain activation across the whole time period. (b) Dynamic brain activation at different time points. In general, the brain activation involved was very weak. A slight increase in $\mathrm{HbO}$ concentration at channel 3 (the left SFG) started at $2 \mathrm{~s}$, continued for a couple of seconds till $4 \mathrm{~s}$, when the decline started. The decrease continued to $10 \mathrm{~s}$ and very little activity can be observed after $10 \mathrm{~s}$. Activation also happened in channels 13 to 14 (the left IFG including the Broca's area), where an increase in $\mathrm{HbO}$ concentration started at $10 \mathrm{~s}$, continued to $15 \mathrm{~s}$, and then started to decrease again.

from 4 to $10 \mathrm{~s} .{ }^{62,63}$ Then, the left IFG covering the Broca's area (channels 13-14) started to dominate the brain activity from 10 to $16 \mathrm{~s}$, indicating that effort in this region was mainly devoted to the production of speech during this period of time. By contrast, unlike the transphrasing task, the pairing task elicited brain activation in the left IFG right after the stimuli were presented. Specifically, as shown in Fig. 7, we can see that the activation was only localized in the Broca's area (channels 13 and 14), which sustained the whole neural response period, with the highest $\mathrm{HbO}$ concentration change at around $5 \mathrm{~s}$. These results suggested that pairing might be taking a "shortcut" since it only involves the Broca's area, which was activated right after the stimuli were presented. ${ }^{8,12,13}$

Figure 8 demonstrated the dynamic brain activation patterns associated with the nontranslation task, indicating that brain activations were mostly identified in the left SFG (channel 3) and left IFG (channels 13-14). More importantly, the results shown in Figs. 5, 6, and 8 exhibited that nontranslation task elicited the lowest $\mathrm{HbO}$ concentration changes and the smallest activation regions. Consequently, our neural findings supported the hypothesis that nontranslation is the most economic strategy for SI. ${ }^{17,64}$ Nontranslation elicited very little activation in the left frontal cortex while both pairing and transphrasing induced enhanced brain activation in the region.

In addition, it is noted that the population of professional simultaneous interpreters is extremely small. The sample size of this study is on par with those of experimental studies involving professional simultaneous interpreters/trainee simultaneous interpreters published in recent years. ${ }^{2,5,65,66}$ Importantly, it has been revealed that training and experience in SI can lead to anatomical and functional changes in the adult brain., ${ }^{2,56-68}$ For the present study, we did our best to make sure that all subjects matched in age, education background, language background, and expertise in simultaneous interpreters. Also, as the stimuli 


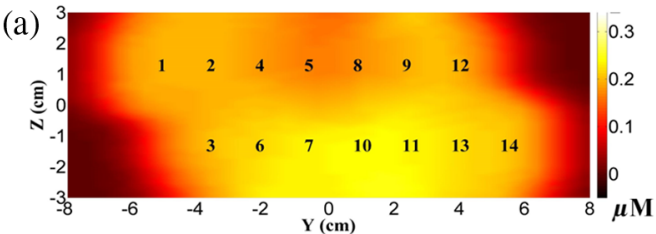

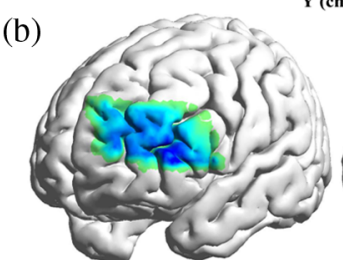

$1 s$

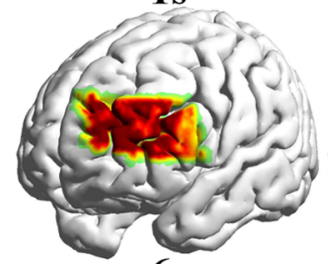

$6 \mathrm{~s}$

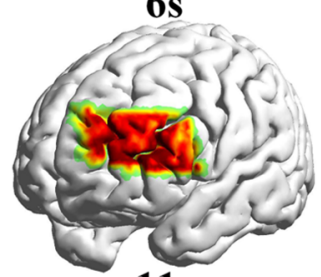

$11 \mathrm{~s}$

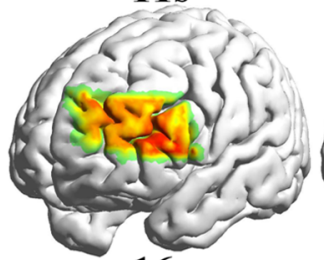

$16 \mathrm{~s}$

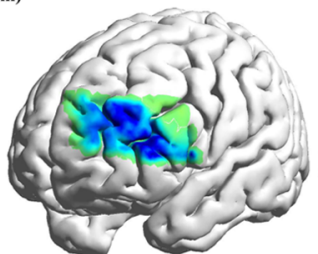

$2 s$

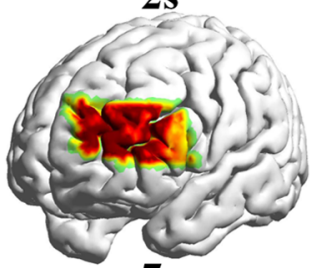

$7 \mathrm{~s}$

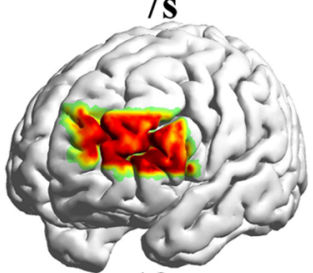

$12 \mathrm{~s}$

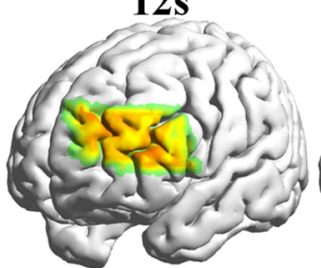

$17 \mathrm{~s}$

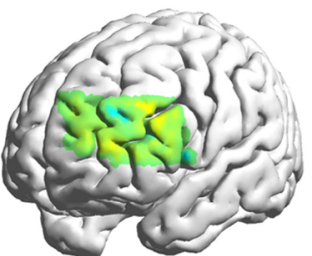

$3 s$

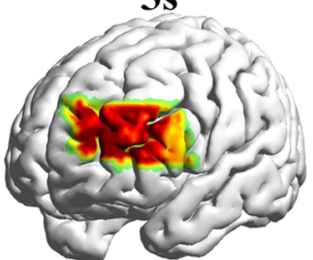

$8 s$

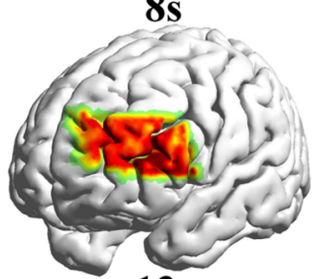

$13 s$

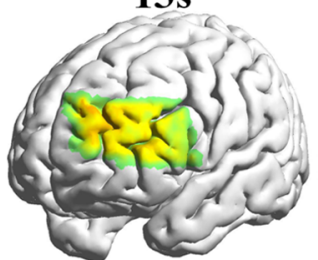

$18 \mathrm{~s}$

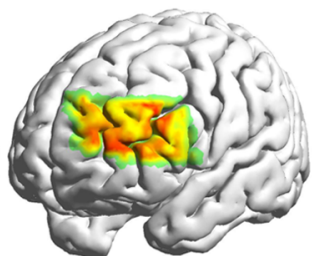

$4 s$

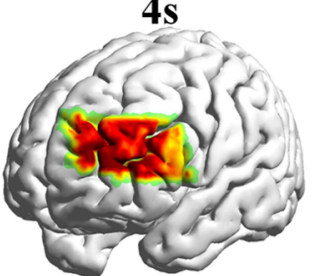

9s

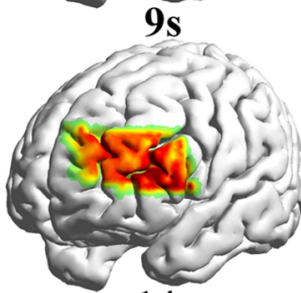

$14 s$

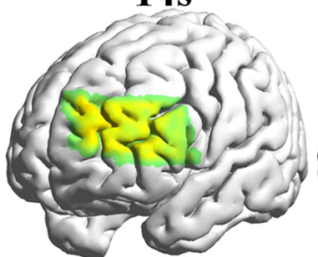

19s

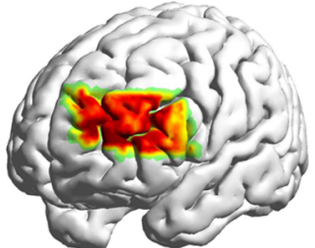

$5 s$

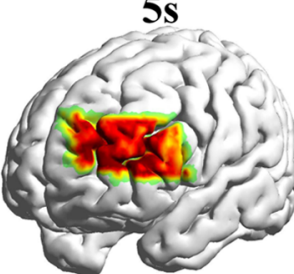

$10 \mathrm{~s}$

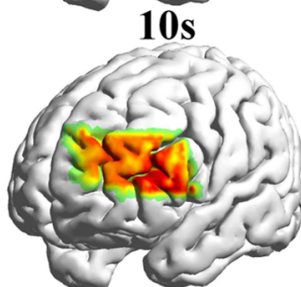

$\boldsymbol{\mu} \mathbf{M}$

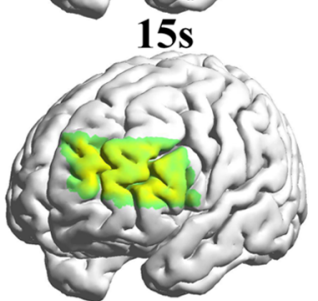

$20 \mathrm{~s}$

Fig. 9 Brain activation map demonstrating changes in brain activity over time ( 1 to $20 \mathrm{~s}$ ) associated with transphrasing stimuli. (a) Averaged brain activation across the whole stimuli period. (b) Dynamic brain patterns at selected time points. We discovered that the whole area of interest, except for the region covered by channels 13 and 14 (the Broca's area), started to have increased $\mathrm{HbO}$ concentration at $3 \mathrm{~s}$. The growth continued till $7 \mathrm{~s}$, when the decline started, and the decrease continued all the way till $20 \mathrm{~s}$. The highest level of $\mathrm{HbO}$ concentration change was recorded in channels 10 (the left MFG) and 11(the left IFG) at 6 to $7 \mathrm{~s}$. HbO concentration peaked in channel 3 (the left SFG), channel 6 (the left SFG), channel 7 (the left MFG), and channel 10 (the left MFG) at 6 to $7 \mathrm{~s}$. HbO concentration in channels 13 to 14 covering the Broca's area started to increase at $10 \mathrm{~s}$ and started to decrease at $16 \mathrm{~s}$.

for the experiment were culture-specific items, steps were taken to make sure that all subjects had been exposed to the same culture. The subjects were selected from among a group of $33 \mathrm{MA}$ students taking the SI course at UM between 2015 and 2016. Only those who achieved Grade A- and above through constant assessment throughout the half-year course - 11 in total were selected for the experimental tests. Track records since 2010 suggested that students of that level were able to provide professional SI service satisfactorily. In fact, half of those selected for the experiments were already professional in-house/freelance simultaneous interpreters. One of the 11 interpreters who took part in the fNIRS experiment was identified as an outlier and was excluded because a postexperiment interview revealed that she had gone through significantly intense training in SI than other subjects, and it is evident that her attainment was much higher.
Consistent with previous findings, $8,12,13,64$ our fNIRS study revealed that transphrasing is the most costly strategy in SI because it induced the most extensive and overall the strongest activation in the brain regions investigated (the left PFC). Behavior results also showed that transphrasing had the lowest accuracy in translation performance. Pairing, on the other hand, induced the most intense, but highly localized activation in the broca's region, and it is evident that nontranslation involved very little processing effort and emerged as the "effort-reduction strategy." It is noted that although rendering by pairing is swift, the translation product is short and usually only contains one disyllabic word in English, the activation it elicited in the Broca's area was even stronger than that from transphrasing within the same brain region. On the other hand, nontranslation, which also produced disyllabic items in the TL, elicited much weaker activation in the Broca's area. These results supported 
the reports that the functions of Broca's area are more than language production - they also play a crucial role in domain-general cognitive control, ${ }^{46-48}$ including the control of interference. Consistent with our hypothesis, pairing requires the highest level of cognitive control among the three strategies while "nontranslation" is the least costly in terms of the energy it consumes for the cognitive control.

Interestingly, the results also imply that in terms of the functional neuroanatomy of language, transphrasing, which involves conceptual mediation, may take the "long route" while pairing takes the "shortcut" linking translation equivalents stored in long-term memory together-a hypothesis proposed by neurolinguists decades ago ${ }^{8,12}$ and is certainly worth further investigations using neuroimaging technologies. Previous work also showed that the right hemisphere is involved in decoding in the native language. ${ }^{20,69}$ To capture evidence for the existence of the "long route," which involves decoding in the native language, it is also important to explore the right IFG region in the future.

In addition, it is noted that motion artifacts can generate significant effect on the quality of fNIRS signals. A bunch of schemes have been developed to resolve this issue, such as principle component analysis, spline interpolation, Kalman filtering, wavelet filtering, and correlation-based signal improvement. Previous studies showed that it is always better to correct for motion artifacts than reject trials and that wavelet filtering is the most effective in correcting this type of artifact. $^{70}$ Meanwhile, it should be pointed out that the absence of short channels in the used probes might affect the analysis accuracy of hemodynamic responses due to the possibility for loss of retrieving spatial functional information in the brain. The biggest challenge for incorporating more channels with short source-detector distance lies in the difficulties in quantifying the accurate differential path length factor by using $\mathrm{CW}$ fNIRS system. It is expected that the short channels should be counted for future fNIRS neuroimaging studies. More importantly, it is also very hard to measure the oxygen saturation and blood flow/blood volume of brain if only analysis of $\mathrm{HbO}$ signals is performed. Further analysis should incorporate both the $\mathrm{HbR}$ and $\mathrm{HbO}$ data to infer the brain activation associated with various cognitive tasks and disorders.

In summary, the present study will pave a path for better understanding the neural mechanism of SI by exploring the regional activation patterns of brain in terms of hemodynamic responses.

\section{Disclosures}

The authors declare no competing financial interests.

\section{Acknowledgments}

This study was supported by MYRG2016-00110-FHS, MYRG2015-00036-FHS, MYRG2015-00150-FAH, MYRG 2016-00096-FAH, and MYRG2017-00139-FAH grants from the University of Macau, Macao SAR, and FDCT 026/2014/ A1 and FDCT 025/2015/A1, grants from the Macao government.

\section{References}

1. B. J. Diamond and G. M. Shreve, "Neural and physiological correlates of translation and interpreting in the bilingual brain," in Translation and Cognition, S. M. Gregory and E. Angelone, Eds., pp. 289-321, John Benjamins, Amsterdam/Philadelphia (2010).
2. M. Becker et al., "Simultaneous interpreters vs. professional multilingual controls: group differences in cognitive control as well as brain structure and function," Neuroimage 134, 250-260 (2016).

3. R. Setton, "Neurolinguistic and cognitive aspects of interpreting," in The Encyclopedia of Applied Linguistics, C. A. Chapelle, Eds., Blackwell Publishing Ltd., Oxford (2013).

4. B. Moser-Mercer, "The search for neuro-physiological correlates of expertise in interpreting," in Translation and Cognition, S. M. Gregory and E. Angelone, Eds., pp. 263-287, John Benjamins, Amsterdam/Philadelphia (2010).

5. A. Hervais-Adelman et al., "fMRI of simultaneous interpretation reveals the neural basis of extreme language control," Cereb. Cortex 25(12), 4727-4739 (2015).

6. A. M. De Groot and I. K. Christoffels, "Language control in bilinguals: monolingual tasks and simultaneous interpreting," Bilingualism: Lang. Cogn. 9(2), 189-201 (2006).

7. A. G. Hervais-Adelman, B. Moser-Mercer, and N. Golestani, "Executive control of language in the bilingual brain: integrating the evidence from neuroimaging to neuropsychology," Front. Psychol. 2, 234 (2011).

8. M. Paradis, "Toward a neurolinguistic theory of simultaneous translation: the framework," Int. J. Psycholinguist. 10(3), 319-335 (1994).

9. M. Paradis, M.-C. Goldblum, and R. Abidi, "Alternate antagonism with paradoxical translation behavior in two bilingual aphasic patients," Brain Lang. 15(1), 55-69 (1982).

10. F. Fabbro, B. Gran, and L. Gran, "Hemispheric specialization for semantic and syntactic components of language in simultaneous interpreters," Brain Lang. 41(1), 1-42 (1991).

11. J. F. Kroll and E. Stewart, "Category interference in translation and picture naming: evidence for asymmetric connections between bilingual memory representations," J. Mem. Lang. 33(2), 149-174 (1994).

12. A. M. De Groot, "The cognitive study of translation and interpretation: three approaches," in Cognitive Processes in Translation and Interpretation, J. H. Danks, Eds., pp. 25-26, Sage Publications, Thousand Oaks, California (1997).

13. A. M. De Groot, Bilingual Cognition: An Introduction, Psychology Press, New York (2011).

14. M. Paradis, "Linguistic, psycholinguistic, and neurolinguistic aspects of "interference" in bilingual speakers: the activation threshold hypothesis," Int. J. Psycholinguist. 9(2), 133-145 (1993).

15. H. Jiang, "The interpreter's glossary in simultaneous interpreting: a survey," Interpreting 15(1), 74-93 (2013).

16. D. D. Resh, "Toward a Byzantine definition of metaphrasis," Greek Roman Byz. Stud. 55(3), 754-787 (2015).

17. D. Gile, Basic Concepts and Models for Interpreter and Translator Training, John Benjamins, Amsterdam/Philadelphia (2009).

18. R. Setton, Simultaneous Interpretation: A Cognitive-Pragmatic Analysis, John Benjamins, Amsterdam/Philadelphia (1999).

19. D. Gile, "Les noms propres en interprétation simultanée," MultilinguaJ. Cross-Cult. Interlang. Commun. 3(2), 79-86 (1984).

20. F. Fabbro et al., "Cerebral lateralization in simultaneous interpretation," Brain Lang. 39(1), 69-89 (1990)

21. V. Darò and F. Fabbro, "Verbal memory during simultaneous interpretation: effects of phonological interference," Appl. Linguist. 15(4), 365381 (1994)

22. W. P. Isham, "Memory for sentence form after simultaneous interpretation: evidence both for and against deverbalization" in Bridging the Gap: Empirical Research in Simultaneous Interpretation, L. B. Moser-Mercer, Eds., pp. 191-211, John Benjamins, Amsterdam (1994).

23. Y. He, "A fresh cognitive perspective to horizontal translation," J. Transl. Stud. 10(1), 77-90 (2007).

24. D. W. Green, "Mental control of the bilingual lexico-semantic system," Bilingualism: Lang. Cogn. 1(2), 67-81 (1998).

25. D. W. Green, "Control, activation, and resource: a framework and a model for the control of speech in bilinguals," Brain Lang. 27(2), 210-223 (1986).

26. K. J. Friston, "Functional and effective connectivity in neuroimaging: a synthesis," Hum. Brain Mapp. 2(1-2), 56-78 (1994).

27. J. Ferreira Duarte, "The politics of non-translation: a case study in Anglo-Portuguese relations," Traduct. Terminol. Rédact. 13(1), 95-112 (2000). 
28. C. Soares and F. Grosjean, "Bilinguals in a monolingual and a bilingual speech mode: the effect on lexical access," Mem. Cogn. 12(4), 380-386 (1984).

29. A. Costa and M. Santesteban, "Lexical access in bilingual speech production: evidence from language switching in highly proficient bilinguals and L2 learners," J. Mem. Lang. 50(4), 491-511 (2004).

30. J. Abutalebi and D. Green, "Bilingual language production: the neurocognition of language representation and control," J. Neurolinguist. 20(3), 242-275 (2007).

31. J. O. Rinne et al., "The translating brain: cerebral activation patterns during simultaneous interpreting," Neurosci. Lett. 294(2), 85-88 (2000).

32. V. Quaresima et al., "Lateral frontal cortex oxygenation changes during translation and language switching revealed by non-invasive near-infrared multi-point measurements," Brain Res. Bull. 59(3), 235-243 (2002).

33. D. Klein et al., "The neural substrates underlying word generation: a bilingual functional-imaging study," Proc. Natl. Acad. Sci. U. S. A. 92(7), 2899-2903 (1995).

34. L. A. Petitto et al., "The "Perceptual Wedge Hypothesis" as the basis for bilingual babies' phonetic processing advantage: new insights from fNIRS brain imaging," Brain Lang. 121(2), 130-143 (2012).

35. F. M. Branzi et al., "Language control in bilinguals: monitoring and response selection," Cereb. Cortex 26(6), 2367-2380 (2015).

36. A. Rodriguez-Fornells, R. De Diego Balaguer, and T. F. Münte, "Executive control in bilingual language processing," Lang. Learn. 56(s1), 133-190 (2006).

37. H. Uylings et al., "Broca's language area from a neuroanatomical and developmental perspective," in The Neurocognition of Language, Colin M. Brown and P. Hagoort, Eds., pp. 319-336, Oxford University Press, Oxford (1999).

38. A. Friederici, "Phonological processing in language production: time course of brain activity," Neuroreport 14(16), 2031-2033 (2003).

39. A. D. Friederici et al., "The role of left inferior frontal and superior temporal cortex in sentence comprehension: localizing syntactic and semantic processes," Cereb. Cortex 13(2), 170-177 (2003).

40. A. D. Friederici et al., "Processing linguistic complexity and grammaticality in the left frontal cortex," Cereb. Cortex 16(12), 1709-1717 (2006).

41. F. Binkofski and G. Buccino, "Motor functions of the Broca's region," Brain Lang. 89(2), 362-369 (2004).

42. N. Nishitani et al., "Broca's region: from action to language," Physiology 20(1), 60-69 (2005).

43. L. Fadiga, L. Craighero, and A. D'Ausilio, "Broca's area in language, action, and music," Ann. N. Y. Acad. Sci. 1169(1), 448-458 (2009).

44. G. Hickok et al., "The role of Broca's area in speech perception: evidence from aphasia revisited," Brain Lang. 119(3), 214-220 (2011).

45. C. J. Price, D. W. Green, and R. Von Studnitz, "A functional imaging study of translation and language switching," Brain 122(12), 22212235 (1999).

46. J. M. Novick, J. C. Trueswell, and S. L. Thompson-Schill, "Broca's area and language processing: evidence for the cognitive control connection," Lang. Linguist. Compass 4(10), 906-924 (2010).

47. S. Elmer, "Broca pars triangularis constitutes a "hub" of the languagecontrol network during simultaneous language translation," Front. Hum. Neurosci. 10, 491 (2016).

48. E. Fedorenko, J. Duncan, and N. Kanwisher, "Language-selective and domain-general regions lie side by side within Broca's area," Curr. Biol. 22(21), 2059-2062 (2012).

49. R. Beurskens et al., "Age-related changes in prefrontal activity during walking in dual-task situations: a fNIRS study," Int. J. Psychophysiol. 92(3), 122-128 (2014).

50. S. Debener et al., "How about taking a low-cost, small, and wireless EEG for a walk?" Psychophysiology 49(11), 1617-1621 (2012).

51. Z. Yuan, "Combining independent component analysis and Granger causality to investigate brain network dynamics with fNIRS measurements," Biomed. Opt. Express 4(11), 2629-2643 (2013).

52. Z. Yuan, "Spatiotemporal and time-frequency analysis of functional near infrared spectroscopy brain signals using independent component analysis," J. Biomed. Opt. 18(10), 106011 (2013).
53. Z. Yuan and J. Ye, "Fusion of fNIRS and fMRI data: identifying when and where hemodynamic signals are changing in human brains," Front. Hum. Neurosci. 7, 676 (2013).

54. X. Lin et al., "Optical mapping of prefrontal activity in pathological gamblers," Appl. Opt. 56(21), 5948-5953 (2017).

55. H. F.-H. Ieong and Z. Yuan, "Abnormal resting-state functional connectivity in the orbitofrontal cortex of heroin users and its relationship with anxiety: a pilot fNIRS study," Sci. Rep. 7, 46522 (2017).

56. S. Tak et al., "Quantification of CMRO2 without hypercapnia using simultaneous near-infrared spectroscopy and fMRI measurements," Phys. Med. Biol. 55(11), 3249-3269 (2010).

57. A. K. Singh et al., "Spatial registration of multichannel multi-subject fNIRS data to MNI space without MRI," Neuroimage 27(4), 842-851 (2005).

58. T. J. Huppert et al., "HomER: a review of time-series analysis methods for near-infrared spectroscopy of the brain," Appl. Opt. 48(10), D280D298 (2009).

59. M. Cope and D. T. Delpy, "System for long-term measurement of cerebral blood and tissue oxygenation on newborn infants by near infra-red transillumination," Med. Biol. Eng. Comput. 26(3), 289-294 (1988).

60. J. Zhang et al., "Mapping the small-world properties of brain networks in deception with functional near-infrared spectroscopy," Sci. Rep. 6, 25297 (2016).

61. A. K. Singh and I. Dan, "Exploring the false discovery rate in multichannel NIRS," Neuroimage 33(2), 542-549 (2006).

62. D. A. Copland et al., "Dopaminergic neuromodulation of semantic processing: a 4-T FMRI study with levodopa," Cereb. Cortex 19(11), 2651-2658 (2009).

63. X. Wang et al., "The neural correlates of the interaction between semantic and phonological processing for Chinese character reading," Front. Psychol. 7, 947 (2016).

64. A. K.-F. Cheung, "Code-mixing and simultaneous interpretation training," Interpret. Newsl. 11, 57-62 (2001).

65. S. Elmer, J. Hänggi, and L. Jäncke, "Processing demands upon cognitive, linguistic, and articulatory functions promote grey matter plasticity in the adult multilingual brain: insights from simultaneous interpreters," Cortex 54, 179-189 (2014).

66. E. Van de Putte et al., "Anatomical and functional changes in the brain after simultaneous interpreting training: a longitudinal study," Cortex 99, 243-257 (2018).

67. Y. Dong and F. Zhong, "Interpreting experience enhances early attentional processing, conflict monitoring and interference suppression along the time course of processing," Neuropsychologia 95, 193-203 (2017).

68. A. Hervais-Adelman et al., "Cortical thickness increases after simultaneous interpretation training," Neuropsychologia 98, 212-219 (2017).

69. T. V. Chernigovskaya, L. J. Balonov, and V. L. Deglin, "Bilingualism and brain functional asymmetry," Brain Lang. 20(2), 195-216 (1983).

70. S. Brigadoi et al., "Motion artifacts in functional near-infrared spectroscopy: a comparison of motion correction techniques applied to real cognitive data," Neuroimage 85, 181-191 (2014).

Xiaohong Lin is a doctoral student in the Faculty of Health Sciences at University of Macau. Her research is focused on cognitive neurosciences.

Victoria Lai Cheng Lei is an associate professor in the Faculty of Arts and Humanities at University of Macau. Her research is focused on translation studies.

Defeng Li is a full professor in the Faculty of Arts and Humanities at University of Macau, and he is also the director of the Centre for Study of Translation-Interpreting and Cognitionin. His research is focused on translation studies and second language education.

Zhen Yuan is an associate professor in the Faculty of Health Sciences with the University of Macau. His research is focused on neurosciences, neuroimaging, biomedical optics, and optical molecular imaging. 